\title{
Circulating ANGPTL8 levels and risk of kidney function decline: Results from the $4 \mathrm{C}$ Study
}

\author{
Huajie Zou ${ }^{1,2+}$, Yongping $\mathrm{Xu}^{1,2+}$, Xiaoyu Meng ${ }^{1,2}$, Danpei Li ${ }^{1,2}$, Xi Chen ${ }^{1,2}$, Tingting Du ${ }^{1,2}$, Yan Yang ${ }^{1,2}$, \\ Yong Chen ${ }^{1,2}$, Shiying Shao ${ }^{1,2}$, Gang Yuan ${ }^{1,2}$, Xinrong Zhou ${ }^{1,2}$, Shuhong Hu ${ }^{1,2}$, Wentao He ${ }^{1,2}$, Delin Ma ${ }^{1,2}$, \\ Junhui Xie ${ }^{1,2}$, Benping Zhang ${ }^{1,2}$, Jianhua Zhang ${ }^{1,2}$, Wenjun $\mathrm{Li}^{3}$, Zhelong Liu ${ }^{1,2^{*}+}$ and Xuefeng $\mathrm{Yu}^{1,2^{*}+}$ (D)
}

\begin{abstract}
Background: ANGPTL8, an important regulator of lipid metabolism, was recently proven to have additional intracellular and receptor-mediated functions. This study aimed to investigate circulating levels of ANGPTL8 and its potential association with the risk of kidney function decline in a cohort study.

Methods: We analysed 2,311 participants aged 40 years old and older from the China Cardiometabolic Disease and Cancer Cohort (4C) Study. Kidney function decline was defined as an estimated glomerular filtration rate (eGFR) less than $60 \mathrm{~mL}$ per minute per $1.73 \mathrm{~m}^{2}$ of body surface area, a decrease in eGFR of $\geq 30 \%$ from baseline, chronic kidney disease (CKD)-related hospitalization or death, or end-stage renal disease. The association between baseline ANGPTL8 levels and kidney function decline was assessed using multivariable-adjusted Cox proportional hazards models, and inverse possibility of treatment weight (IPTW) was utilized to prevent overfitting.

Results: There were 136 (5.9\%) cases of kidney function decline over a median of 3.8 years of follow-up. We found that serum ANGPTL8 levels at baseline were elevated in individuals with kidney function decline compared to those without kidney function decline during follow-up (718.42 \pm 378.17 vs. $522.04 \pm 283.07 \mathrm{pg} / \mathrm{mL}, \mathrm{p}<0.001)$. Compared with the first quartile, multivariable-adjusted hazard ratio (95\% confidence intervals [Cls]) for kidney function decline was $2.59(95 \% \mathrm{Cl}, 1.41-4.77)$ for the fourth ANGPTL8 quartile. Furthermore, compared with patients in the first ANGPTL8 quartile, those in the fourth ANGPTL8 quartile were more likely to report a higher stage of CKD (relative risk: $1.33 ; 95 \% \mathrm{Cl}, 1.01-1.74)$. The conclusions of the regression analyses were not altered in the IPTW models. Multivariable-adjusted restricted cubic spline analyses suggested a linear relationship of ANGPTL8 with kidney function decline ( $p$ for nonlinear trend $=0.66$, $p$ for linear trend $<0.001$ ).
\end{abstract}

Conclusions: Participants with higher circulating ANGPTL8 levels were at increased risk for kidney function decline, highlighting the importance of future studies addressing the pathophysiological role of ANGPTL8 in CKD.

Keywords: ANGPTL8, Kidney function decline, eGFR, 4C Study

*Correspondence: liuzhelong@163.com; xfyu188@163.com

${ }^{\dagger}$ Huajie Zou, Yongping Xu, Zhelong Liu and Xuefeng Yu contributed equally to this work

${ }^{1}$ Division of Endocrinology, Department of Internal Medicine, Tongji Hospital, Tongji Medical College, Huazhong University of Science and Technology, 1095 Jiefang Avenue, Wuhan 430030, China

Full list of author information is available at the end of the article

\section{Background}

Angiopoietin-like protein 8 (ANGPTL8) has been called different names, such as betatrophin, TD26, "refeeding induced in fat and liver" (RIFL), lipasin, and PRO1185 [1]. In the blood, ANGPTL8 forms a complex with ANGPTL3 or ANGPTL4 to inhibit lipoprotein lipase

c) The Author(s) 2021. This article is licensed under a Creative Commons Attribution 4.0 International License, which permits use, sharing, adaptation, distribution and reproduction in any medium or format, as long as you give appropriate credit to the original author(s) and the source, provide a link to the Creative Commons licence, and indicate if changes were made. The images or other third party material in this article are included in the article's Creative Commons licence, unless indicated otherwise in a credit line to the material. If material is not included in the article's Creative Commons licence and your intended use is not permitted by statutory regulation or exceeds the permitted use, you will need to obtain permission directly from the copyright holder. To view a copy of this licence, visit http://creativeco mmons.org/licenses/by/4.0/. The Creative Commons Public Domain Dedication waiver (http://creativecommons.org/publicdomain/ zero/1.0/) applies to the data made available in this article, unless otherwise stated in a credit line to the data. 
(LPL) and regulate triglyceride (TG) metabolism under fed or fasted conditions, respectively [2]. Recently, evidence has emerged for additional intracellular and receptor-mediated functions of ANGPTL8, with implications in NF-kB-mediated inflammation, autophagy, adipogenesis, intracellular lipolysis and regulation of the circadian clock [3]. Elevated levels of plasma ANGPTL8 are associated with metabolic syndrome [45, 46], type 2 diabetes mellitus (T2DM) [4-6], non-alcoholic fatty liver disease (NAFLD)/non-alcoholic steatohepatitis (NASH) [7, 8], atherosclerosis $[9,10]$ and hypertension [11]. Furthermore, hypertension and atherosclerosis are important risk factors for future estimated glomerular filtration rate (eGFR) decline and the development of kidney disease $[12,13]$.

Chronic kidney disease (CKD) is a devastating condition that is reaching epidemic levels owing to the increasing prevalence of diabetes mellitus, hypertension and obesity, as well as the ageing of the population [14]. CKD may progress to irreversible nephron loss, end-stage renal disease and/or premature death [14]. The burden of CKD is substantial. According to WHO global health estimates, CKD accounted for 12.2 deaths per 100,000 people, ranking fourteenth in the list of leading causes of death [15].

Previous cross-sectional studies revealed that ANGPTL8 levels were increased in T2DM patients with albuminuria $[16,17]$. Our previous study also found that circulating ANGPTL8 levels were negatively correlated with the eGFR [18], which indicates that ANGPTL8 may play a potential role in the progression of kidney disease. Whether ANGPTL8 is a protective regulator or a risk factor in kidney disease remains to be explored. In this work, we retrospectively investigated the association between circulating ANGPTL8 levels and the risk for developing CKD in a 5-year cohort study.

\section{Materials and methods Study design and population}

Participants in the present study were recruited from Hubei Province, China, from 2011 to 2012 as part of the Risk Evaluation of Cancer in Chinese Diabetic Individuals: A Longitudinal (REACTION) Study, which was renamed the China Cardiometabolic Disease and Cancer Cohort (4C) Study [19-22]. The study is a multicentre, population-based, prospective cohort study of Chinese adults aged $\geq 40$ years investigating the associations of glucose homeostasis with clinical outcomes, including diabetes, cardiovascular disease (CVD), cancer, and allcause mortality [23].

Of the 10,999 study participants, 9,221 (83.8\%) were followed up during 2011-2016. As shown in Additional file 1: Fig. S1, we excluded participants who were older than 90 years $(\mathrm{N}=47)$, had prevalent $\mathrm{CKD}$ $\left(\right.$ eGFR $<60 \mathrm{~mL} / \mathrm{min} / 1.73 \mathrm{~m}^{2}$ or CKD-related hospitalization) $(\mathrm{N}=245)$ or missing serum creatinine values at baseline $(\mathrm{N}=979)$, were not fasting at baseline $(\mathrm{N}=1,031)$ or visits $(\mathrm{N}=508)$, were missing body mass index (BMI) values, glucose measurements, glycaemic measures, lipid profiles or information on a history of CVD, diabetes or hypertension $(\mathrm{N}=3,142)$, or had missing outcome data $(\mathrm{N}=935)$. Additionally, the highest and lowest $0.5 \%$ of ANGPTL8 values were trimmed $(\mathrm{N}=23)$ [23], leaving 2,311 participants for analysis.

The Committee on Human Research at Ruijin Hospital, Shanghai Jiaotong University School of Medicine, approved the study protocol (Shanghai, China: 14/2004), and all of the participants provided written informed consent. All of the methods were performed in accordance with the relevant guidelines and regulations.

\section{Laboratory tests of ANGPTL8}

Blood samples were collected after overnight fasting. Serum was separated by centrifugation, aliquoted and then stored at $-80^{\circ} \mathrm{C}$. Fasting serum ANGPTL8 levels were assessed using ELISA kits (Eiaab Science, Wuhan, China; Catalogue No. E11644h) with an intra-assay coefficient of variation $(\mathrm{CV})$ of $\leq 6.5 \%$ and an interassay $\mathrm{CV}$ of $\leq 9.2 \%$ (provided by the manufacturer). The procedures were performed in accordance with the manufacturer's instructions. All of the samples were analysed in duplicate.

\section{Outcome assessment}

Kidney function decline was defined as meeting at least 1 of the following criteria: (1) eGFR $<60 \mathrm{~mL} / \mathrm{min} / 1.73$ $\mathrm{m}^{2}$ according to the CKD-Epidemiology Collaboration (CKD-EPI) formula [24, 25], (2) a decrease in eGFR of $\geq 30 \%$ from baseline, established as a surrogate for the development of kidney failure [26], (3) hospitalization related to CKD stage $\geq 3$, (4) death related to CKD stage $\geq 3$, or (5) end-stage renal disease (ESRD). The CKD stage was classified based on the eGFR category, which was assigned as follows: (1) G1, eGFR $\geq 90 \mathrm{~mL} / \mathrm{min} / 1.73$ $\mathrm{m}^{2}$; (2) $\mathrm{G} 2,60 \leq \mathrm{eGFR}<90 \mathrm{~mL} / \mathrm{min} / 1.73 \mathrm{~m}^{2}$; (3) G3, $30 \leq$ eGFR $<60 \mathrm{~mL} / \mathrm{min} / 1.73 \mathrm{~m}^{2}$; (4) G4, $15 \leq \mathrm{eGFR}<30 \mathrm{~mL} /$ $\mathrm{min} / 1.73 \mathrm{~m}^{2}$; and (5) G5, eGFR $<15 \mathrm{~mL} / \mathrm{min} / 1.73 \mathrm{~m}^{2}[25$, 27]. All of these outcomes were confirmed by death certificates and hospital records.

\section{Statistical analysis}

Continuous variables are expressed as the means \pm standard deviations (SDs) (for normally distributed data) or medians (interquartile ranges, IQRs) (for asymmetrically distributed data). Normal distribution of the data was tested using the 
Kolmogorov-Smirnov test. We obtained $\mathrm{p}$ values using the Kruskal-Wallis test for continuous variables and the $\chi^{2}$ test for categorical variables. We used Cox proportional hazards models to investigate the association between ANGPTL8 levels at baseline and the risk of kidney function decline. The relationship between ANGPTL8 and CKD stage was tested using ordinal logistic regression. We adjusted multivariable models for baseline age, sex, BMI, high-density lipoprotein (HDL), low-density lipoprotein (LDL), TC (total cholesterol), TGs, aspartate aminotransferase (AST), alanine transaminase (ALT), and history of diabetes, hypertension and CVD. The inverse possibility of treatment weight (IPTW) was utilized to prevent overfitting [28]. Stratified analyses were conducted according to age, sex, BMI, diabetes, hypertension, CVD or hyperlipidaemia at baseline. Correlations between variables were assessed using Spearman and partial correlation analyses. Potential nonlinear relationships between the levels of ANGPTL8 and the incidence of clinical outcomes were examined with restricted cubic splines [29]. A knot was located at the 25th, 50th, and 75th percentiles for ANGPTL8. Tests for nonlinearity were conducted using likelihood ratio tests. If a test for nonlinearity was not significant, we conducted a test for linearity. Receiver-operator characteristic (ROC) curves were drawn, and the performance of the model was evaluated by the area under the curve (AUC). A 2-tailed $p$ value $<0.05$ was considered significant. SPSS software version 20.0, Stata software version 12.0, and SAS were used for all of the analyses.

\section{Results}

\section{Baseline characteristics}

Of the 2,311 participants, 136 (5.9\%) patients developed kidney function decline during a median follow-up of 3.8 years (Table 1). We found that serum ANGPTL8 levels at baseline were elevated in individuals with kidney function decline compared with those without kidney function decline during the follow-up (718.42 \pm 378.17 vs. $522.04 \pm 283.07 \mathrm{pg} / \mathrm{mL}, \mathrm{p}<0.001$, Fig. 1).

The baseline characteristics of the included participants according to quartiles of ANGPTL8 are presented in Table 1. Age, sex, fasting plasma glucose (FPG), $2 \mathrm{~h}$ plasma glucose concentration (2 h PG), HDL, TG, AST, ALT, creatinine, and eGFR at baseline changed with ANGPTL8 levels (all $\mathrm{p}$ values $<0.05$ ). Furthermore, the prevalence of hypertension, hyperlipidaemia and diabetes increased in the highest quartile of ANGPTL8 levels $(\mathrm{p}<0.05)$. There was no significant difference in medication use for patients with diabetes, hypertension or hyperlipidaemia (all $\mathrm{p}$ values $>0.05$, Additional file 1 : Table S1).

\section{ANGPTL8 correlated with kidney function decline}

As shown in Table 1, increasing quartiles of ANGPTL8 levels were associated with elevated incidences of kidney function decline and an increased number of patients with a higher CKD stage (all p values $<0.001$ ). Compared with the first quartile, multivariable-adjusted hazard ratios (HRs) (95\% confidence intervals [CIs]) for kidney function decline were 2.59 (95\% CI, 1.41-4.77; model 3 in Table 2) and 1.73 (95\% CI, 1.27-2.36; IPTW model in Table 2) for the fourth ANGPTL8 quartile. Furthermore, a per-SD increase in ANGPTL8 levels was associated with a $31 \%$ higher risk for kidney function decline (HR, 1.31; 95\% CI, 1.13-1.51). Additional file 1: Table S2 shows the associations between ANGPTL8 and CKD stage. Adjusted estimates revealed that compared with participants in the first ANGPTL8 quartile, those in the fourth ANGPTL8 quartile were more likely to report a higher stage of CKD (relative risk [RR]: 1.33; 95\% CI, 1.01-1.74; model 3 in Additional file 1: Table S2), which was consistent with the results in the IPTW model.

After controlling for multiple variables, ANGPTL8 levels were also positively correlated with age $(r=0.24)$, FPG $(r=0.07)$, TGs $(r=0.04)$, AST $(r=0.08)$ and creatinine $(r=0.13)$ and inversely correlated with HDL $(r=-0.05)$, eGFR at baseline $(r=-0.13)$, eGFR at the final visit $(r=$ $-0.11)$ and eGFR change $(\Delta$ eGFR\%) $(r=-0.07)$ (all $\mathrm{p}$ values $<0.05$; Additional file 1 : Table S3).

Multivariable-adjusted restricted cubic spline analyses suggested a linear relationship of ANGPTL8 with the HR of kidney function decline ( $\mathrm{p}$ for nonlinear trend $=0.66, \mathrm{p}$ for linear trend $<0.001$; Fig. 2).

The ROC curve shown in Additional file 1: Fig. S2 depicts the performance of ANGPTL8 concentration in detecting kidney function decline. The optimal cut-off point was $608.20 \mathrm{pg} / \mathrm{mL}$. Using this cut-off value, diagnostic efficiency for kidney function decline reached the highest value: the AUC was 0.66 (95\% CI, 0.61-0.71, $\mathrm{p}<0.001$ ), with sensitivity and specificity of $55.1 \%$ and $70.1 \%$, respectively.

\section{Stratified analyses}

We performed stratified analyses according to age, sex, BMI, diabetes, hypertension, CVD or hyperlipidaemia at baseline. As shown in Fig. 3, the association between ANGPTL8 levels and kidney function decline showed increased significance in individuals with $\mathrm{BMI} \geq 24 \mathrm{~kg} /$ $\mathrm{m}^{2}$, older age ( $\geq 60$ years), hypertension and no CVD (all $\mathrm{p}$ value $<0.05$ ). However, the association was diminished in individuals without hypertension or with CVD (all $\mathrm{p}$ value $>0.05$ ). 
Table 1 Clinical and biochemical parameters for participants, according to quartile of ANGPTL8 levels

\begin{tabular}{|c|c|c|c|c|c|c|}
\hline Characteristics & Q1 (187.78-287.78) & Q2 (359.06-436.95) & Q3 (517.94-605.04) & Q4 (751.47-1057.66) & All & $P$ value \\
\hline N & 577 & 579 & 578 & 577 & 2311 & \\
\hline Age (years) & $58(52-64)$ & $60(53-67)$ & $63(56-69)$ & $64(58-71)$ & $62(55-68)$ & $<0.001$ \\
\hline Male (\%) & $135(23.4)$ & $194(33.5)$ & $240(41.5)$ & $261(45.2)$ & 830 (35.9) & $<0.001$ \\
\hline BMI $\left(\mathrm{kg} / \mathrm{m}^{2}\right)$ & $23.77(21.67-25.94)$ & $23.34(21.47-26.14)$ & $23.23(21.30-25.68)$ & $23.24(21.17-25.64)$ & $23.43(21.41-25.81)$ & 0.09 \\
\hline WHR & $0.87(0.83-0.90)$ & $0.87(0.83-0.90)$ & $0.87(0.83-0.90)$ & $0.88(0.83-0.91)$ & $0.87(0.83-0.90)$ & 0.33 \\
\hline HbA1c (\%) & $5.70(5.40-6.00)$ & $5.70(5.40-6.00)$ & $5.70(5.50-6.10)$ & $5.70(5.40-6.20)$ & $5.70(5.40-6.00)$ & 0.07 \\
\hline FPG (mmol/L) & $5.27(4.92-5.74)$ & $5.22(4.88-5.71)$ & $5.34(4.93-6.02)$ & $5.38(4.94-6.15)$ & $5.28(4.92-5.91)$ & $<0.001$ \\
\hline $2 \mathrm{~h} \mathrm{PG}(\mathrm{mmol} / \mathrm{L})$ & $6.18(5.19-7.65)$ & $6.32(5.23-7.68)$ & $6.50(5.44-8.44)$ & $6.70(5.55-9.12)$ & $6.43(5.30-8.16)$ & $<0.001$ \\
\hline $\mathrm{HDL}(\mathrm{mmol} / \mathrm{L})$ & $1.48(1.26-1.74)$ & $1.44(1.25-1.70)$ & $1.47(1.24-1.73)$ & $1.40(1.17-1.69)$ & $1.45(1.23-1.71)$ & 0.006 \\
\hline LDL (mmol/L) & $2.82(2.30-3.37)$ & $2.77(2.27-3.32)$ & $2.80(2.26-3.33)$ & $2.79(2.29-3.35)$ & $2.79(2.28-3.34)$ & 0.88 \\
\hline TG (mmol/L) & $1.22(0.87-1.66)$ & $1.14(0.81-1.65)$ & $1.08(0.84-1.63)$ & $1.31(0.88-1.92)$ & $1.19(0.85-1.73)$ & $<0.001$ \\
\hline TC (mmol/L) & $5.02(4.35-5.59)$ & $4.94(4.34-5.58)$ & $4.96(4.38-5.64)$ & $5.04(4.32-5.66)$ & $4.99(4.35-5.62)$ & 0.64 \\
\hline ALT (U/L) & $12(9-17)$ & $12(9-16)$ & $12(10-17)$ & $14(10-19)$ & $13(10-17)$ & $<0.001$ \\
\hline AST (U/L) & $20(17-24)$ & $21(18-25)$ & $21(18-26)$ & $23(19-28)$ & $21(18-26)$ & $<0.001$ \\
\hline $\begin{array}{l}\text { Creatinine at baseline } \\
(\mu \mathrm{mol} / \mathrm{L})\end{array}$ & $58.50(54.70-63.45)$ & $59.40(55.00-64.60)$ & $60.60(56.10-65.80)$ & $62.30(56.85-68.30)$ & $60.30(55.50-65.50)$ & $<0.001$ \\
\hline $\begin{array}{l}\text { eGFR at baseline (mL/ } \\
\left.\min / 1.73 \mathrm{~m}^{2}\right)\end{array}$ & $99.77(94.23-105.04)$ & $98.52(93.35-104.31)$ & 96.72 (91.72-102.07) & $94.95(89.45-100.35)$ & 97.36 (92.19-103.05) & $<0.001$ \\
\hline Hypertension (\%) & $300(52.0)$ & $301(52.0)$ & $330(57.1)$ & $341(59.1)$ & $1272(55.0)$ & 0.03 \\
\hline Hyperlipidaemia (\%) & $213(36.9)$ & $213(36.8)$ & $211(36.5)$ & $260(45.1)$ & $897(38.8)$ & 0.005 \\
\hline DM (\%) & $114(19.8)$ & $102(17.6)$ & $153(26.5)$ & $172(29.8)$ & $541(23.4)$ & $<0.001$ \\
\hline CVD (\%) & $27(4.7)$ & $30(5.2)$ & $19(3.3)$ & $33(5.7)$ & $109(4.7)$ & 0.24 \\
\hline $\begin{array}{l}\text { Kidney function decline } \\
(\%)\end{array}$ & $16(2.8)$ & $29(5.0)$ & $25(4.3)$ & $66(11.4)$ & $136(5.9)$ & $<0.001$ \\
\hline \multicolumn{7}{|l|}{ CKD stage } \\
\hline G1 (\%) (eGFR $\geq 90)$ & $382(66.2)$ & $355(61.3)$ & $332(57.4)$ & $253(43.8)$ & $1322(57.2)$ & $<0.001$ \\
\hline $\mathrm{G} 2(\%)(60 \leq \mathrm{eGFR}<90)$ & $183(31.7)$ & $201(34.7)$ & $229(39.6)$ & $274(47.5)$ & $887(38.4)$ & \\
\hline $\mathrm{G} 3(\%)(30 \leq \mathrm{eGFR}<60)$ & $11(1.9)$ & $22(3.8)$ & $16(2.8)$ & $47(8.1)$ & $96(4.2)$ & \\
\hline G4 (\%) $(15 \leq \mathrm{eGFR}<30)$ & $1(0.2)$ & $1(0.2)$ & $1(0.2)$ & $2(0.3)$ & $5(0.2)$ & \\
\hline G5 (\%) $(\mathrm{eGFR}<15)$ & $0(0)$ & $0(0)$ & $0(0)$ & $1(0.2)$ & $1(0.04)$ & \\
\hline
\end{tabular}

BMI body-mass index, WHR waist hip rate, HbA1c glycated haemoglobin A1c, FPG fasting plasma glucose, $2 \mathrm{~h} P G 2 \mathrm{~h}$ plasma glucose concentration, $H D L$ high density lipoprotein, $L D L$ low density lipoprotein, TG triglycerides, TC total cholesterol, ALT alanine transaminase, $A S T$ aspartate aminotransferase, eGFR glomerular filtration rate, $D M$ diabetes mellitus, CVD cardiovascular diseases, $C K D$ chronic kidney disease

\section{Discussion}

\section{ANGPTL8 levels correlated with kidney function decline}

This retrospective cohort study found that serum baseline ANGPTL8 levels were elevated and independently associated with kidney function decline. Furthermore, multivariable-adjusted restricted cubic spline suggested a dose-response relationship of ANGPTL8 with the risk for kidney function decline. However, estimates for this association in individuals with CVD or without hypertension were not statistically significant.

ANGPTL8, an important regulator of TG metabolism, has recently been proven to have additional intracellular and receptor-mediated functions [3, 30-32]. Previous studies found that ANGPTL8 levels were increased in diabetic nephropathy and associated with eGFR [16, 17, 33, 34]. However, whether ANGPTL8 exerts an effect on the progression of nephropathy or whether it responds to renal dysfunction remains an open question. Nevertheless, our longitudinal study investigated whether ANGPTL8 may be a potential risk factor for CKD.

\section{Potential mechanisms for the association}

The underlying mechanisms for these pathophysiological associations await exposure. Our study found that ANGPTL8 was positively correlated with age, FPG, AST, and TG but inversely correlated with HDL. Previous studies found that ANGPTL8 levels were positively related to age $[10,18,35]$. Stratified analyses also showed that the association between ANGPTL8 levels and kidney function decline was more significant in older individuals (age $\geq 60$ years). Age is related to mitochondrial dysfunction, increased oxidative stress, inflammation, 


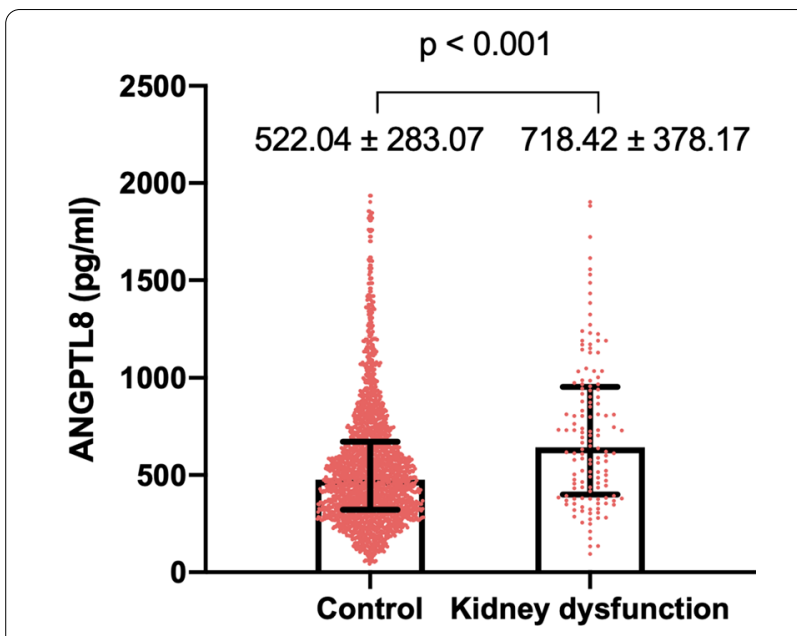

Fig. 1 Circulating ANGPTL8 levels in patients with and without kidney function decline during follow-up. The boxplot displays median and IQR

and hormonal changes [47], which play an important role in the pathogenesis of CKD progression [14]. Second, the accumulation of lipids in the kidney was shown to promote the progression of renal tubular damage and interstitial fibrosis by leading to inflammation, reactive oxygen species (ROS) production and endogenous electrical stress [36]. Thus, dysregulated lipid metabolism may be the potential mechanism underlying the involvement of ANGPTL8 in the development of CKD. Furthermore, NAFLD was reported to be a driver of CKD [37, 38]. ANGPTL8, a novel hepatokine, was increased in NAFLD [7] and positively correlated with AST. Therefore, ANPTL8 may be a potential link between NAFLD and CKD. Finally, ANGPTL8 has been shown to negatively regulate NF- $\mathrm{KB}$, a key transcription factor implicated in the inflammatory signalling cascade [32]. However, circulating levels of ANGPTL8 have been reported to be elevated in diseases associated with inflammation, such as T2DM [4-6], atherosclerosis [10], and NASH [8]. In addition, plasma levels of ANGPTL8 have been found to be elevated in patients with severe infections, and a strong correlation between circulating ANGPTL8 and the lipopolysaccharide-induced acute inflammatory response in animal models has been observed [32]. Thus, Abu-Farha et al. suggested that even though intracellular ANGPTL8 may prevent the activation of NF- $\mathrm{kB}$, the extracellular actions of circulating ANGPTL8 may cause inflammation [3], which plays a key role in CKD. However, estimates of the association between ANGPTL8 and kidney function decline in individuals with CVD or without hypertension were not statistically significant. The null association in patients with CVD may be due to the relatively small number of events, small sample size (13/109) and correspondingly low power, although there was a trend showing that patients with CVD had a decline in kidney function in numbers similar to patients without CVD. Hypertension status is an important risk factor for future eGFR decline and the development of

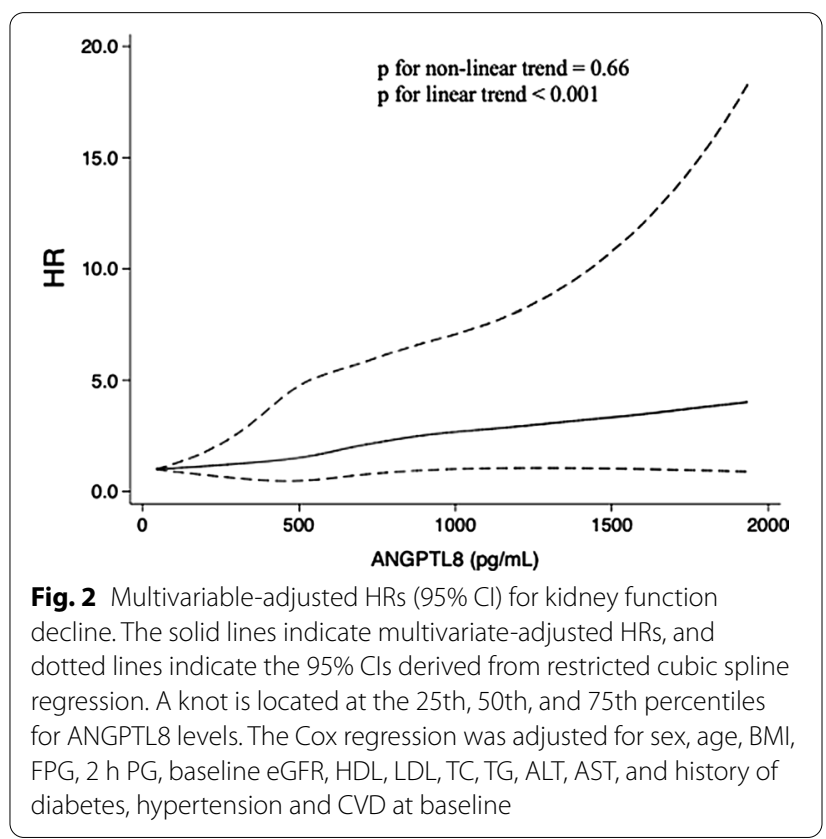

Table 2 Relation between ANGPTL8 and kidney function decline, according to quartiles of ANGPTL8

\begin{tabular}{lllr}
\hline ANGPTL8 & Model 1 & Model 2 & Model 3 \\
\hline Q1 (Reference) & 1 & 1 & 1 \\
Q2 (HR, 95\% Cl) & $1.91(1.04-3.51)$ & $1.86(0.98-3.54)$ & $1.52(0.79-2.94)$ \\
Q3 (HR, 95\% Cl) & $1.74(0.93-3.25)$ & $1.45(0.74-2.85)$ & $1.43(0.73-2.80)$ \\
Q4 (HR, 95\% Cl) & $4.66(2.70-8.05)$ & $3.99(2.21-7.20)$ & $1.72(1.10-2.68)$ \\
Per-SD increase & $1.64(1.45-1.85)$ & $1.55(1.36-1.77)$ & $1.36(0.86-2.14)$ \\
\hline
\end{tabular}

Model 1 was unadjusted

Model 2 was adjusted for age, sex and BMI

Model 3 was adjusted for all variables in model 2 plus baseline eGFR, HDL, LDL, TC, TG, ALT, AST, history of diabetes, hypertension and CVD at baseline $H R$ hazards ratio, $C l$ confidence intervals, IPTW inverse possibility of treatment weight, SD standard deviation 


\section{Subgroup}

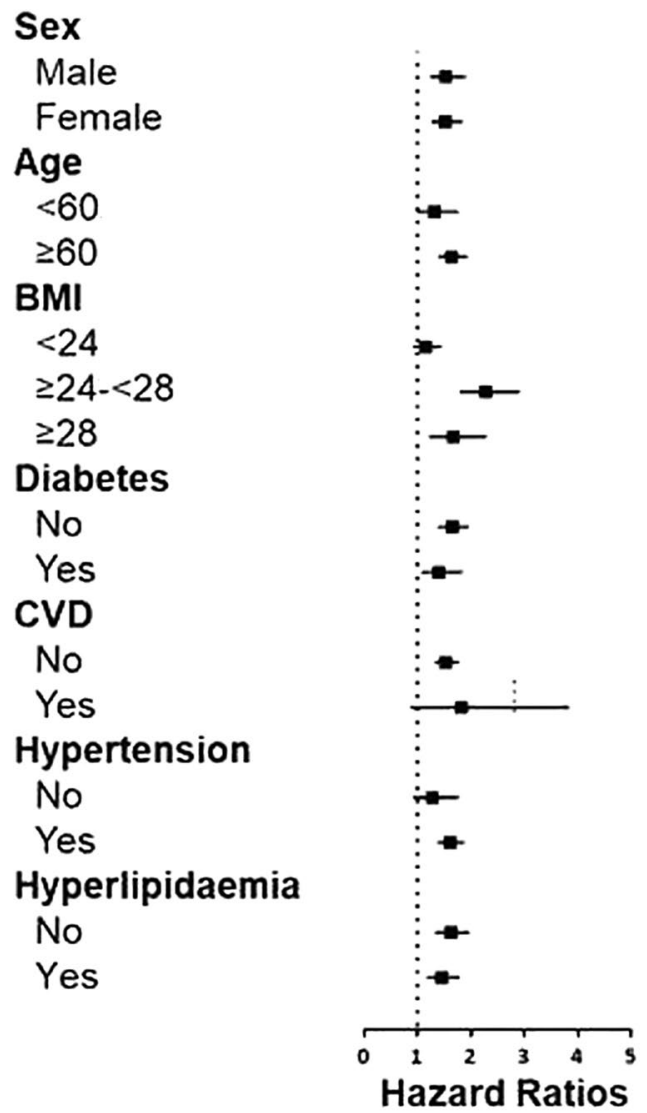

\section{N Case $\mathrm{HR}(95 \% \mathrm{Cl})$}

$\begin{array}{ccc}830 & 69 & 1.53(1.23-1.91) \\ 1481 & 67 & 1.52(1.25-1.85) \\ & & \\ 987 & 39 & 1.32(0.98-1.77) \\ 1324 & 97 & 1.64(1.39-1.94) \\ & & \\ 1294 & 70 & 1.16(0.92-1.46) \\ 776 & 43 & 2.28(1.79-2.92) \\ 241 & 18 & 1.67(1.21-2.30) \\ 1770 & 95 & 1.65(1.37-1.97) \\ 541 & 41 & 1.40(1.07-1.85) \\ & & \\ 2202 & 123 & 1.53(1.32-1.78) \\ 109 & 13 & 1.82(0.86-3.85) \\ & & \\ 1039 & 34 & 1.28(0.92-1.77) \\ 1272 & 102 & 1.61(1.37-1.88) \\ 1414 & 71 & 1.63(1.33-1.98) \\ 897 & 65 & 1.45(1.17-1.80)\end{array}$

Fig. 3 Stratified analyses of the HR of kidney function decline according to a 1-SD increase in ANGPTL8. The final model adjusted for age, sex, BMI, FPG, 2 h PG, HDL, LDL, TC, TG, ALT, AST, and history of diabetes, hypertension and CVD at baseline, except the strata variable

kidney disease [13], which may be due to higher intraglomerular pressure and progressive arteriosclerosis [12, 39, 40]. Elevated ANGPTL8 levels have been reported to be associated with atherosclerosis $[9,10]$ and hypertension [11]. Therefore, ANGTPTL8 may contribute to the acceleration of the atherogenic process and renal artery stenosis in patients with hypertension, leading to eGFR decline and the development of CKD.

On the other hand, ANGPTL8 shares considerable structural similarity with ANGPTL3 and ANGPTL4 [41]. As the structure generally determines the function, ANGPTL8 may play a similar role to ANGPTL4. Previous studies suggested that ANGPTL4 is a critical link between proteinuria and hypertriglyceridaemia [42]. Furthermore, podocytes secrete ANGPTL4 and mediate proteinuria in glucocorticoid-sensitive nephrotic syndrome [43]. Therefore, we speculated that podocytes or other kidney cells may also secrete ANGPTL8 in a pathological state and be involved in the pathogenesis of CKD. Above all, further experiments are needed to elucidate the pathophysiological significance of this finding.

\section{Limitations}

There are some limitations of this study. First, all of the participants in our study were Chinese adults aged $\geq 40$ years, limiting the generalizability of the findings. Interestingly, the previous 4 cross-sectional studies enrolled populations with a mean age $>50$ years and concluded that elevated ANGPTL8 levels were associated with kidney function decline $[16,17,33$, $34]$, especially in diabetic patients [16, 17, 33]. Among the above studies, 2 were conducted in Asian populations [16, 17], 1 was conducted in an European population [34] and 1 was conducted in an African population [33]. ANGPTL8 levels were reported to vary over a 150fold range and differed between racial/ethnic groups 
(Blacks $>$ Hispanics $>$ Whites) [44]. Therefore, the association should be confirmed in other ethnic and age groups. Second, the retrospective design might also have carried the risk of selection bias; therefore, prospective studies are needed to confirm the results. Regardless, this study is the first longitudinal study showing that ANGPTL8 could be a potential risk factor for CKD. Third, we did not have albuminuria data. Therefore, we cannot properly address the association between ANGPTL8 and urinary albumin excretion or the albumin/creatinine ratio as additional markers for kidney function. Finally, the relatively short follow-up duration reduced the number of clinical events and the study's statistical power, especially in stratified analyses. Future studies should be conducted with larger sample sizes to verify the findings of our study.

\section{Conclusion}

In conclusion, participants with higher circulating ANPTL8 levels had an increased risk for kidney function decline, highlighting the importance of future studies addressing the pathophysiological role of ANGPTL8 in CKD.

\begin{abstract}
Abbreviations
ALT: Alanine transaminase; ANGPTL8: Angiopoietin-like protein 8; AST: Aspartate aminotransferase; BMI: Body mass index; Cl: Confidence intervals; CKD: Chronic kidney disease; CV: Coefficient of variation; CVD: Cardiovascular disease; DM: Diabetes mellitus; eGFR: Estimated glomerular filtration rate; ESRD: End-stage renal disease; FPG: Fasting plasma glucose; $2 \mathrm{~h} \mathrm{PG:} 2 \mathrm{H}$ plasma glucose concentration; HbA1c: Glycated haemoglobin A1 c; HR: Hazard ratios; HOMA- $\beta$ : Homeostasis model assessment of $\beta$ cell function; HOMA-IR: Homeostasis model assessment of insulin resistance; HDL: High-density lipoprotein; IPTW: Inverse possibility of treatment weight; IQR: Interquartile range; NAFLD: Non- alcoholic fatty liver disease; NASH: Non-alcoholic steatohepatitis; LDL: Low-density lipoprotein; RR: Relative risk; SD: Standard deviation; TC: Total cholesterol; TG: Triglycerides; T2DM: Type 2 diabetes mellitus; WHR: Waist-hip ratio.
\end{abstract}

\section{Supplementary Information}

The online version contains supplementary material available at https://doi. org/10.1186/s12933-021-01317-3.

Additional file 1: Table S1. Medication for patients with chronic diseases, according to quartile of ANGPTL8 levels. Table S2. Ordinal logistic regression analysis of the association between ANGPTL8 and renal stage. Table S3. Correlations between ANGPTL8 levels and clinical variables. Figure S1. Flow diagram for the study population selection. Figure S2. ROC curves for the performance of ANGPTL8 concentration in detecting kidney function decline.

\section{Acknowledgements}

We would like to thank Dr. Zhangping Li in the clinical laboratory of the Division of Endocrinology for their contributions in collecting and handling samples.

\section{Authors' contribution}

Study design: $X Y$ and $Z L$; data acquisition: $H Z, Y X, X C, T D, Y Y, S S, G Y, S H$, and WH; laboratory tests: $\mathrm{HZ}, \mathrm{XM}, \mathrm{DL}$, and JZ; data analyses: $\mathrm{HZ}, \mathrm{YX}, \mathrm{DM}$, and $\mathrm{YC}$; data interpretation: all authors. Each author contributed important intellectual content during manuscript drafting or revision and accepts accountability for the overall work by ensuring that questions pertaining to the accuracy or integrity of any portion of the work are appropriately investigated and resolved. All authors read and approved the final manuscript.

\section{Funding}

This study was supported by the National Natural Science Foundation of China $(81974109,81570740)$ and grants from the National Key R\&D Program of China (2016YFC0901203).

\section{Availability of data and materials}

The datasets used and/or analysed during the current study are available from the corresponding author on reasonable request.

\section{Declarations}

Ethics approval and consent to participate

The Committee on Human Research at Ruijin Hospital, Shanghai Jiaotong University School of Medicine, approved the study protocol (Shanghai, China: 14/2004), and all of the participants provided written informed consent.

\section{Consent for publication}

Not applicable.

\section{Competing interests}

The authors declare no conflicts of interest.

\section{Author details}

${ }^{1}$ Division of Endocrinology, Department of Internal Medicine, Tongji Hospital, Tongji Medical College, Huazhong University of Science and Technology, 1095 Jiefang Avenue, Wuhan 430030, China. ${ }^{2}$ Branch of National Clinical Research Center for Metabolic Diseases, Hubei, China. ${ }^{3}$ Computer Center, Tongji Hospital, Tongji Medical College, Huazhong University of Science and Technology, Wuhan, China.

Received: 22 March 2021 Accepted: 7 June 2021

Published online: 24 June 2021

\section{References}

1. Zhang R, Abou-Samra AB. A dual role of lipasin (betatrophin) in lipid metabolism and glucose homeostasis: Consensus and controversy. Cardiovasc Diabetol. 2014;13:133.

2. Zhang R. The ANGPTL3-4-8 model, a molecular mechanism for triglyceride trafficking. Open Biol. 2016;6(4):150272.

3. Abu-Farha M, Ghosh A, Al-Khairi I, Madiraju SRM, Abubakr J, Prentki M. The multi-faces of Angptl8 in health and disease: Novel functions beyond lipoprotein lipase modulation. Prog Lipid Res. 2020;80:101067.

4. Chen X, Lu P, He W, Zhang J, Liu L, Yang Y, et al. Circulating betatrophin levels are increased in patients with type 2 diabetes and associated with insulin resistance. J Clin Endocrinol Metab. 2015;100:E96-100.

5. Hu H, Sun W, Yu S, Hong X, Qian W, Tang B, et al. Increased circulating levels of betatrophin in newly diagnosed type 2 diabetic patients. Diabetes Care. 2014;37:2718-22.

6. Lu P, Chen X, Zhang Z, Zhang J, Yang Y, Liu Z, et al. Insulin upregulates betatrophin expression via PI3K/Akt pathway. Sci Rep. 2017;7:5594.

7. García-Monzón C, Petrov PD, Rey E, Marañón P, del Pozo-Maroto E, Guzmán C, et al. Angiopoietin-like protein 8 is a novel vitamin D receptor target gene involved in nonalcoholic fatty liver pathogenesis. Am J Pathol. 2018;188:2800-10.

8. Lee YH, Lee SG, Lee CJ, Kim SH, Song YM, Yoon MR, et al. Association between betatrophin/ANGPTL8 and non-alcoholic fatty liver disease: Animal and human studies. Sci Rep. 2016;6:24013.

9. Jiao X, Yang Y, Li L, Yu H, Yang Y, Li J, et al. Angiopoietin-like protein 8 accelerates atherosclerosis in ApoE-/- mice. Atherosclerosis. 2020;307:63-71.

10. Zheng T, Ge B, Liu H, Chen B, Qin L, Xiao L, et al. Triglyceride-mediated influence of serum angiopoietin-like protein 8 on subclinical 
atherosclerosis in type 2 diabetic patients: Results from the GDMD study in China. Cardiovasc Diabetol. 2018;17:84.

11. Abu-Farha M, Cherian P, Qaddoumi MG, AlKhairi I, Sriraman D, Alanbaei M, et al. Increased plasma and adipose tissue levels of ANGPTL8/Betatrophin and ANGPTL4 in people with hypertension. Lipids Health Dis. 2018;17:35.

12. Drüeke TB, Massy ZA. Atherosclerosis in CKD: Differences from the general population. Nat Rev Nephrol. 2010;6(12):723-35.

13. Yu Z, Rebholz CM, Wong E, Chen Y, Matsushita K, Coresh J, et al. Association Between Hypertension and Kidney Function Decline: The Atherosclerosis Risk in Communities (ARIC) Study. Am J Kidney Dis. 2019;74(3):310-9.

14. Ruiz-Ortega M, Rayego-Mateos S, Lamas S, Ortiz A, Rodrigues-Diez RR Targeting the progression of chronic kidney disease. Nat Rev Nephrol. 2020;16(5):269-88.

15. Webster AC, Nagler EV, Morton RL, Masson P. Chronic Kidney Disease. Lancet. 2017;389(10075):1238-52.

16. Chen CC, Susanto H, Chuang WH, Liu TY, Wang CH. Higher serum betatrophin level in type 2 diabetes subjects is associated with urinary albumin excretion and renal function. Cardiovasc Diabetol. 2016;15:3.

17. Yang L, Song J, Zhang X, Xiao L, Hu X, Pan H, et al. Association of serum angiopoietin-like protein 8 with albuminuria in type 2 diabetic patients: Results from the GDMD Study in China. Front Endocrinol (Lausanne). 2018;9:414.

18. Zou H, Chen X, Li D, Li W, Xie J, Shao S, et al. Predictive values of ANGPTL8 on risk of all-cause mortality in diabetic patients: from the REACTION Study. Cardiovasc Diabetol. 2020;19:121.

19. Ning G, Guang N, Shanghai J, Jiajun Z, Yiming M, Chao L, et al. Risk Evaluation of cAncers in Chinese diabeTic Individuals: A IONgitudinal (REACTION) study. J Diab. 2012;4:172-3.

20. Bi Y, Lu J, Wang W, Mu Y, Zhao J, Liu C, et al. Cohort profile: Risk evaluation of cancers in Chinese diabetic individuals: A longitudinal (REACTION) study. J Diab. 2014;6:147-57.

21. Ning G, Bloomgarden Z. Diabetes and cancer: Findings from the REACTION study. J Diabetes. 2015;7:143-4.

22. Lu J, Bi Y, Wang T, Wang W, Mu Y, Zhao J, et al. The relationship between insulin-sensitive obesity and cardiovascular diseases in a Chinese population: Results of the REACTION study. Int J Cardiol. 2014;172:388-94.

23. Lu J, He J, Li M, Tang X, Hu R, Shi L, et al. Predictive value of fasting glucose, postload glucose, and hemoglobin A1c on risk of diabetes and complications in Chinese adults. Diab Care. 2019;42:1539-48.

24. Inker LA, Schmid CH, Tighiouart H, Eckfeldt JH, Feldman HI, Greene T, et al. Estimating Glomerular Filtration Rate from Serum Creatinine and Cystatin C. N Engl J Med. 2012;367:20-9.

25. Stevens PE, Levin A. Kidney Disease: Improving Global Outcomes Chronic Kidney Disease Guideline Development Work Group Members. Evaluation and management of chronic kidney disease: synopsis of the kidney disease: improving global outcomes 2012 clinical practice guideline. Ann Intern Med. 2013;158(11):825-30.

26. Coresh J, Turin TC, Matsushita K, Sang Y, Ballew SH, Appel LJ, et al. Decline in estimated glomerular filtration rate and subsequent risk of end-stage renal disease and mortality. JAMA. 2014;311:2518-31.

27. Inker LA, Astor BC, Fox CH, Isakova T, Lash JP, Peralta CA, et al. KDOQI US commentary on the $2012 \mathrm{KDIGO}$ clinical practice quideline for the evaluation and management of CKD. Am J Kidney Dis. 2014;63(5):713-35.

28. Schuster T, Lowe WK, Platt RW. Propensity score model overfitting led to inflated variance of estimated odds ratios. J Clin Epidemiol. 2016;80:97-106

29. Durrleman S, Simon R. Flexible regression models with cubic splines. Stat Med. 1989;8(5):551-61.
30. Zhang $Y$, Zheng L, Huang $K$. A new way to regulate inflammation: selective autophagic degradation of IKKY mediated by ANGPTL8. Cell Stress. 2018:2:66-8.

31. Chen S, Feng M, Zhang S, Dong Z, Wang Y, Zhang W, et al. Angptl8 mediates food-driven resetting of hepatic circadian clock in mice. Nat Commun. 2019;10:3518.

32. Zhang Y, Guo X, Yan W, Chen Y, Ke M, Cheng C, et al. ANGPTL8 negatively regulates NF-KB activation by facilitating selective autophagic degradation of IKKY. Nat Commun. 2017:8:2164.

33. Issa YA. The potential role of angiopoietin-like protein-8 in type 2 diabetes mellitus : a possibility for predictive diagnosis and targeted preventive measures? EPMA J. 2019;10:239-48.

34. Maurer L, Schwarz F, Fischer-Rosinsky A, Schlueter N, Brachs S, Möhlig M, et al. Renal function is independently associated with circulating betatrophin. PLoS ONE. 2017;12(3):e0173197.

35. Pu D, Li L, Yin J, Liu R, Yang G, Liao Y, et al. Circulating ANGPTL8 Is associated with the presence of metabolic syndrome and insulin resistance in polycystic ovary syndrome young women. Mediators Inflamm. 2019;2019:6321427.

36. Gai Z, Wang T, Visentin M, Kullak-Ublick GA, Fu X, Wang Z. Lipid accumulation and chronic kidney disease. Nutrients. 2019;11:722.

37. Byrne CD, Targher G. NAFLD as a driver of chronic kidney disease. J Hepatol. 2020;72:785-801.

38. Marcuccilli M, Chonchol M. NAFLD and chronic kidney disease. Int J Mol Sci. 2016;17:562.

39. Keane WF, Eknoyan G, Willis K. Proteinuria, albuminuria, risk, assessment, detection, elimination (PARADE): A position paper of the National Kidney Foundation. Am J Kidney Dis. 1999;33(5):1004-10.

40. Yoshioka T, Rennke HG, Salant DJ, Deen WM, Ichikawa I. Role of abnormally high transmural pressure in the permselectivity defect of glomerular capillary wall: A study in early passive Heymann nephritis. Circ Res. 1987;61(4):531-8.

41. Li Y, Teng C. Angiopoietin-like proteins 3, 4 and 8: Regulating lipid metabolism and providing new hope for metabolic syndrome. J Drug Target. 2014;22:679-87.

42. Clement LC, Macé C, Avila-Casado C, Joles JA, Kersten S, Chugh SS. Circulating angiopoietin-like 4 links proteinuria with hypertriglyceridemia in nephrotic syndrome. Nat Med. 2014;20(1):37-46.

43. Clement LC, Avila-casado C, Macé C, Soria E, Bakker WW, Kersten S, et al. Podocyte secreted Angiopoietin-like 4 mediates proteinuria in glucocorticoid sensitive nephrotic syndrome. Nat Med. 2011;17(1):117-22.

44. Oldoni F, Bass K, Kozlitina J, Hudson H, Shihanian LM, Gusarova V, et al. Genetic and Metabolic Determinants of Plasma Levels of ANGPTL8. J Clin Endocrinol Metab. 2021;106(6):1649-67.

45. Crujeiras AB, Zulet MA, Abete I, Amil M, Carreira MC, Martínez JA, et al. Interplay of atherogenic factors, protein intake and betatrophin levels in obese-metabolic syndrome patients treated with hypocaloric diets. Int J Obes (Lond). 2016;40:403-10.

46. Abu-Farha M, Abubaker J, Al-Khairi I, Cherian P, Noronha F, Kavalakatt S, et al. Circulating angiopoietin-like protein 8 (betatrophin) association with HsCRP and metabolic syndrome. Cardiovasc Diabetol. 2016;15:25.

47. Schneider JL, Rowe JH, Garcia-de-Alba C, Kim CF, Sharpe AH, Haigis MC. The aging lung: Physiology, disease, and immunity. Cell. 2021;184(8):1990-2019.

\section{Publisher's Note}

Springer Nature remains neutral with regard to jurisdictional claims in published maps and institutional affiliations. 